\title{
Potencia del salto en jugadores de fútbol sala después de la utilización del rodillo de espuma y la facilitación neuromuscular propioceptiva en la musculatura isquiosural
}

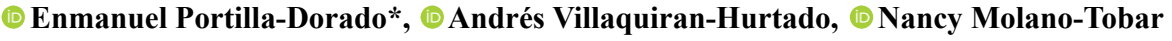 \\ Departamento de Educación Física, Recreación y Deportes, Universidad del Cauca, Popayán, Colombia
}

\begin{abstract}
Resumen
En el entrenamiento deportivo se plantea que, probablemente, la flexibilidad es la capacidad física que menos trabajo exige y cuyos resultados genera mayor controversia. Sin embargo, diversas técnicas de estiramiento se implementan como método para la prevención de lesiones. El objetivo de este estudio fue determinar los cambios en la potencia del salto después de implementar dos programas de entrenamiento de la flexibilidad en deportistas de fútbol sala. Se hizo un estudio experimental con evaluaciones antes y después de la intervención, en el cual se aplicaron dos programas de entrenamiento de la flexibilidad de la musculatura isquiosural mediante la técnica de facilitación neuromuscular propioceptiva y el uso del rodillo de espuma. Las pruebas de salto de los futbolistas participantes se evaluaron con electromiográfíca de los isquiosurales y pruebas de flexibilidad antes y después de la intervención. Se encontró un aumento significativo en la altura del salto con contramovimiento (Counter Movement Jump, CMJ) y del salto Abalakov en el grupo que utilizó el rodillo de espuma, en tanto que con la técnica de facilitación neuromuscular se logró un aumento en los valores del salto Abalakov y en el unipodal derecho en comparación con el grupo de control, con diferencias que presentaban significación estadística $(\mathrm{p} \leq 0,05)$. Se evidenció una disminución en la activación electromiográfíca de los músculos semitendinoso y bíceps femoral derecho durante el salto en cuclillas después de la intervención $(p \leq 0,05)$. En conclusión, una adecuada planificación y estructuración del entrenamiento de la flexibilidad mediante el uso del rodillo de espuma y la facilitación neuromuscular propioceptiva permitieron mejorar la eficiencia de la contracción muscular y la capacidad de salto en deportistas de fútbol sala de Popayán. (C) 2019. Acad. Colomb. Cienc. Ex. Fis. Nat.
\end{abstract}

Palabras clave: Rendimiento deportivo; Estiramientos; Electromiografía; Rodillo de espuma; Potencia; Facilitación neuromuscular propioceptiva.

Jump power in futsal players after foam roller use and proprioceptive neuromuscular facilitation in the ischiosural muscles

\begin{abstract}
In sports training, flexibility is possibly the physical capacity that demands less work and whose results generate the greatest controversy. However, different stretching techniques are implemented as a method for injury prevention. This study sought to determine jump power changes after the implementation of two flexibility training programs in futsal players. We conducted an experimental study with pre- and post-intervention evaluations of two flexibility training programs for the ischiosural muscles using proprioceptive neuromuscular facilitation and the foam roller. The futsal players' jumps were evaluated with electromyography of the ischiosural muscles and flexibility tests before and after the intervention. The results showed a significant increase in height in the countermovement and Abalakov jumps in the group using the foam roller while the neuromuscular facilitation technique resulted in a statistically significant $(\mathrm{p} \leq 0.05)$ increase in the Abalakov and the right unipodal jumps compared to the control group. We found a decrease in the electromyographic activation of the semitendinosus and right femoral biceps muscles during the squat jump after the intervention. In conclusion, adequate planning and structuring of flexibility training with techniques such as the foam roller and the proprioceptive neuromuscular facilitation improved the muscle contraction efficiency and the jumping ability in indoor soccer players from the city of Popayán. (C) 2019. Acad. Colomb. Cienc. Ex. Fis. Nat.
\end{abstract}

Key words: Sports performance; Stretching electromyography; Foam roller; Power; Propioceptive neuromuscular facilitation stretching.

*Correspondencia:

Enmanuel Portilla-Dorado; efportilla@unicauca.edu.co; Recibido: 27 de febrero de 2019; Aceptado: 2 de mayo de 2019; Editor: Luis Fernando García 


\section{Introducción}

El fútbol sala es un deporte de alta intensidad, con movimientos acíclicos variados y de corta duración. Durante un juego, los atletas cambian de actividad cada 3,28 segundos y recorren entre 105 y 160,2 metros por minuto; entre el 20 y el $26 \%$ de dichas distancias se hace con una intensidad superior al $85 \%$ de la frecuencia cardiaca máxima (Rodrigues-Santa Cruz, et al., 2016).

Una característica del juego es la ejecución de sprints máximos repetidos y de corta duración. En los desplazamientos de alta intensidad es posible discriminar los niveles de rendimiento de los jugadores: cuanto mayor es el rendimiento, mayor es la tendencia a que el jugador recorra una distancia mayor en alta intensidad durante el juego (Matzenbacherab, et al., 2014). Arins, et al. (2015) y Carminatti, et al. (2015) hacen referencia a las características fisiológicas del fútbol sala, deporte en el que el sistema adenosintrifosfato - fosfocreatinina (adenosine triphosphate: ATP and phosphocreatine: PC, ATP-PC) es la principal fuente de energía para alcanzar máximos esfuerzos de corta duración.

La capacidad de salto es una de las cualidades más importantes y determinantes en la práctica deportiva y constituye un gesto básico en el deporte que se prioriza en las rutinas de entrenamiento (López, et al., 2015; Picabea \& Yanci, 2015; Aniotz, et al., 2015). El salto como acción multiarticular exige niveles de fuerza con un adecuado control motor y coordinación intramuscular e intermuscular. Los principales músculos que intervienen en el salto vertical son los extensores de rodilla, cadera y tobillo, en cuya ejecución contribuyen aproximadamente con el 49 , el 28 y en $23 \%$, respectivamente (Luarte, et al., 2014).

En este sentido, es importante resaltar que actividades como el salto, el sprint y los cambios de dirección implican una alta intensidad y activación de la musculatura de los miembros inferiores, especialmente de los músculos isquiosurales, que son sometidos a una gran exigencia del ciclo estiramiento-acortamiento, e involucran esfuerzos con gran variedad de movimientos y una elevada concentración de las fibras musculares rápidas, lo que requiriere que el musculo sea lo suficientemente flexible para almacenar y expulsar energía elástica, beneficiando el rendimiento y disminuyendo la posibilidad de sufrir lesiones (Ayala, et al., 2010). Además, la relación entre la potencia muscular y los gestos deportivos del fútbol sala puede verse influenciada por la edad, el entrenamiento, la adaptación previa al evento y los aspectos madurativos (Ogura, et al., 2007).

Es así como en 1999, Bosco, et al. (citado por Dal Pupo, et al., 2016), aseguraron en su estudio que las propiedades neuromusculares del atleta pueden ser mejoradas con el entrenamiento y la adaptación del deportista. Uno de los hechos controvertidos en el entrenamiento y el rendimiento deportivo han sido los beneficios y las contraindicaciones de la práctica de algún método de estiramiento neuromuscular para la potencia del salto. Es claro que la capacidad de salto y de amortiguación del individuo es importante para lograr optimizar el rendimiento en el deporte, y que el poco tiempo que se tiene para entrenar esta habilidad es una problemática común entre los entrenadores, por lo cual el desarrollo de esta cualidad requiere entrenamientos no solo eficaces sino también seguros y realizables en poco tiempo (Ruiz, 2013; Sainz-de-Baranda-Andújar \& Ayala, 2010; Lavandero, et al., 2017).

En algunos estudios se ha registrado un descenso en la capacidad de la contracción máxima voluntaria al realizar algunas técnicas como los estiramientos estáticos y la facilitación neuromuscular propioceptiva (Nelson, et al., 2013), pero en otros no se ha encontrado una disminución en la capacidad de salto vertical después de realizar varios tipos de estiramiento muscular (Ogura, et al., 2007; Woolstenhulme, et al., 2006).

Por otro lado, las actividades de salto, velocidad y cambios de dirección, acciones frecuentes en el fútbol sala, son de alta exigencia para el ciclo de estiramientoacortamiento del músculo, sin embargo, poco se recurre a los programas de entrenamiento, desconociendo que los isquiosurales son músculos poliarticulares con una elevada concentración de fibras musculares rápidas, lo que lleva a que el músculo se acorte y las unidades de músculo tendinosas no sean lo suficientemente flexibles para almacenar y expulsar la gran cantidad de energía elástica que beneficia la contracción muscular (Ayala, et al., 2010).

En este contexto, las técnicas de entrenamiento de la flexibilidad se vienen incorporando a los procesos de desarrollo deportivo, aunque la falta de estudios en la región sobre estas nuevas propuestas, como el uso de la técnica de facilitación neuromuscular propioceptiva y la utilización del rodillo de espuma, ha resultado en una insuficiente evidencia científica sobre su rendimiento en el fútbol sala. Por ello, en este estudio se propuso determinar los cambios en la potencia de los saltos después de la implementación de dos programas de entrenamiento de la flexibilidad en deportistas de fútbol sala.

\section{Materiales y métodos}

Diseño y muestra. Se hizo un estudio experimental, comparativo, con evaluaciones antes y después de la intervención. La muestra se seleccionó de forma aleatoria e incluyó a 23 jugadores masculinos de fútbol sala de un club deportivo participante en el torneo nacional de ascenso de esta disciplina del 2018. La participación en la investigación se ajustó a los siguientes criterios de inclusión: pertenecer y tener el reconocimiento como jugador por parte del club, no presentar ninguna lesión o enfermedad que impidiese la participación durante la intervención, participar de forma voluntaria y firmar el consentimiento informado. Los criterios de exclusión tenidos en cuenta fueron: no cumplir con el $90 \%$ de las sesiones de entrenamiento, no completar las pruebas previas y posteriores a la intervención, el retiro voluntario y presentar lesión o enfermedad durante el programa de intervención. 
Procedimiento. Los objetivos del estudio se compartieron con las directivas, los entrenadores y los deportistas, y estos últimos aceptaron la participación firmando el consentimiento informado. Posteriormente, se hicieron las evaluaciones previas a la intervención, en las cuales se recolectó la información sociodemográfica y los antecedentes deportivos de cada atleta (días de entrenamiento a la semana, horas de entrenamiento por sesión, años de práctica del deporte, posición de juego, dominancia, antecedentes de lesiones y práctica de otra disciplina deportiva) (Álvarez-Pérez \& López-Aguilar, 2018) mediante una encuesta previamente ajustada en una prueba piloto.

Una vez se recolectaron los datos, se evaluó la composición corporal mediante la toma de medidas antropométricas (masa corporal, talla, índice de masa corporal, porcentaje de grasa, sumatoria de pliegues cutáneos) adoptadas por la International Society for the Advancement of Kinanthropometry, ISAK (Stewart, et al., 2011). Para hallar el porcentaje de grasa se utilizó la fórmula de Carter (Rodríguez, 2016), la cual establece que:

porcentaje de grasa $(\%$ grasa $)=2,585+\left(0,1051 * \sum\right.$ pliegue tricipital + pliegue subescapular + pliegue supraespinal + pliegue abdominal + pliegue muslo + pliegue medial pierna.

Posteriormente, se evaluó la potencia del salto mediante la plataforma de contacto AXON JUMP y el programa AXON JUMP 4.0, diseñada para la evaluación de distintas capacidades mecánicas cinemáticas del deportista, entre ellas la capacidad de salto, determinando la altura de cada uno de los saltos verticales (Santiago, et al., 2015; Departamento Administrativo del Deporte, la Recreación, la Actividad Física y el Aprovechamiento del Tiempo Libre - Coldeportes, 2015). Los saltos usados se basan en el protocolo descrito por Bosco, et al. (1983), cuya batería de pruebas de saltos verticales valoran las características funcionales y neuromusculares. Las pruebas realizadas fueron: el test de salto en cuclillas, el de salto de contramovimiento, el test de salto Abalakov, y el test de salto unipodal (derecho e izquierdo).

Cada uno de los saltos se registró con electromiografía de superficie (Myer, et al., 2005) utilizando un electromiógrafo inalámbrico de superficie MYON (http://www.biomec.com. co/Analisis-muscular-myon), con una velocidad de muestreo de $4.000 \mathrm{~Hz}$ por canal, resolución de 12 bits, alcance del transmisor al receptor de hasta $30 \mathrm{~m}$, y frecuencia de transmisión de 2,4 Ghz. El registro se hizo sobre los vientres musculares entre la unión miotendinosa y el punto motor de los músculos semitendinosos y bíceps femoral de manera bilateral, siguiendo las recomendaciones indicadas por la European Recommendations for Surface Electromyography SENIAM project (Hermens, et al., 1999) (Figura 1). La información extraída de las señales mioeléctricas superficiales permitió analizar el comportamiento global de los músculos estudiados y los parámetros cuantitativos; la amplitud fue el parámetro utilizado como indicador de la actividad muscular y se determinó por el valor cuadrático medio (root mean square - RMS).
La valoración se complementó con las pruebas de flexibilidad (Ayala, et al., 2012) de la musculatura isquiosural con las pruebas de sentarse y alcanzar (sit and reach) (Gutiérrez-Manzanedo, et al., 2018) y de elevación de pierna recta (derecha e izquierda) (Sainz-de Baranda, $\boldsymbol{e t}$ al., 2012) utilizando un goniómetro digital y su respectivo programa Unicore (mobee $₫$ med).

Después de la evaluación inicial los participantes se dividieron aleatoriamente en tres grupos para la implementación de los programas de flexibilidad. El grupo número uno realizó estiramientos mediante la técnica de facilitación neuromuscular propioceptiva; el grupo número dos los hizo con la técnica del rodillo de espuma, en tanto que el tercer grupo, que operó como grupo de control, no recibió ningún entrenamiento de flexibilidad.

La intervención se realizó durante ocho semanas, con tres sesiones semanales como lo sugieren Ayala, et al. (2010) para el entrenamiento de la flexibilidad. Los programas se ejecutaban durante el calentamiento de los deportistas previo a la fase central; el estímulo de entrenamiento de la flexibilidad tenía una duración de 15 minutos y un tiempo de ejecución por ejercicio de 30 segundos (Bonjour, 2017); el tiempo de descanso entre los ejercicios y el volumen de entrenamiento para el grupo de estiramientos con el rodillo de espuma (figura 2) se organizó y se planificó siguiendo las recomendaciones de Cheatham, et al., (2015) y de Mohr,

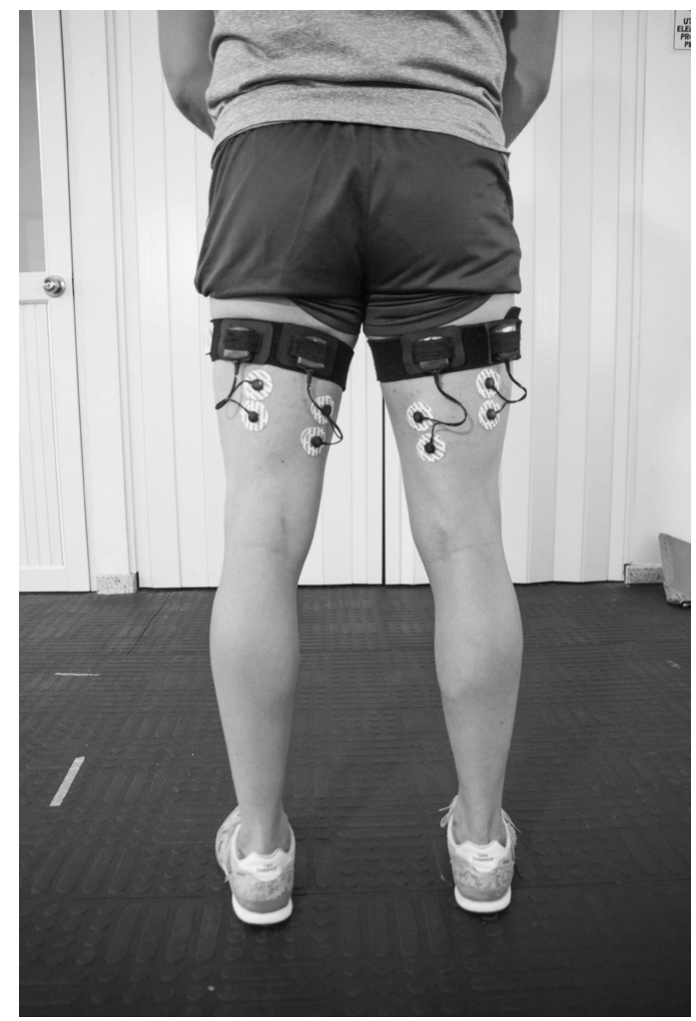

Figura 1. Ubicación de los electrodos para la toma del registro electromiográfico de los músculos isquiosurales durante los saltos verticales 
et al. (2014), así mismo el programa de Facilitación Neuromuscular Propioceptiva se planteó desde la perspectiva de McAtee, R. y Charland (2007), quienes en su libro Estiramientos Facilitados $3^{\mathrm{a}}$ edición, sugieren la intensidad, el tiempo, el volumen y la progresión de la técnica FNP (estiramiento, contracción, estiramiento), como aparece en la figura 3

La progresión semana a semana de los dos programas se efectuó aumentando la intensidad de los ejercicios con base en la percepción subjetiva del esfuerzo medida mediante la escala de Borg modificada por Castañer, et al. (2015), Alberto, et al. (2016) y Foster (1998). Para finalizar el procedimiento se hizo la evaluación posterior a la intervención.

Análisis estadístico. El análisis estadístico se realizó con el programa IBM Statistical Package for the Social Sciences SPSS $®$, versión 23. Se aplicaron pruebas de normalidad y homogeneidad de varianzas (de Shapiro Wilk y de Levene, respectivamente); a las variables que cumplían con la normalidad se les aplicaron la t de Student y la exacta de Fisher para determinar la significación estadística dentro de los

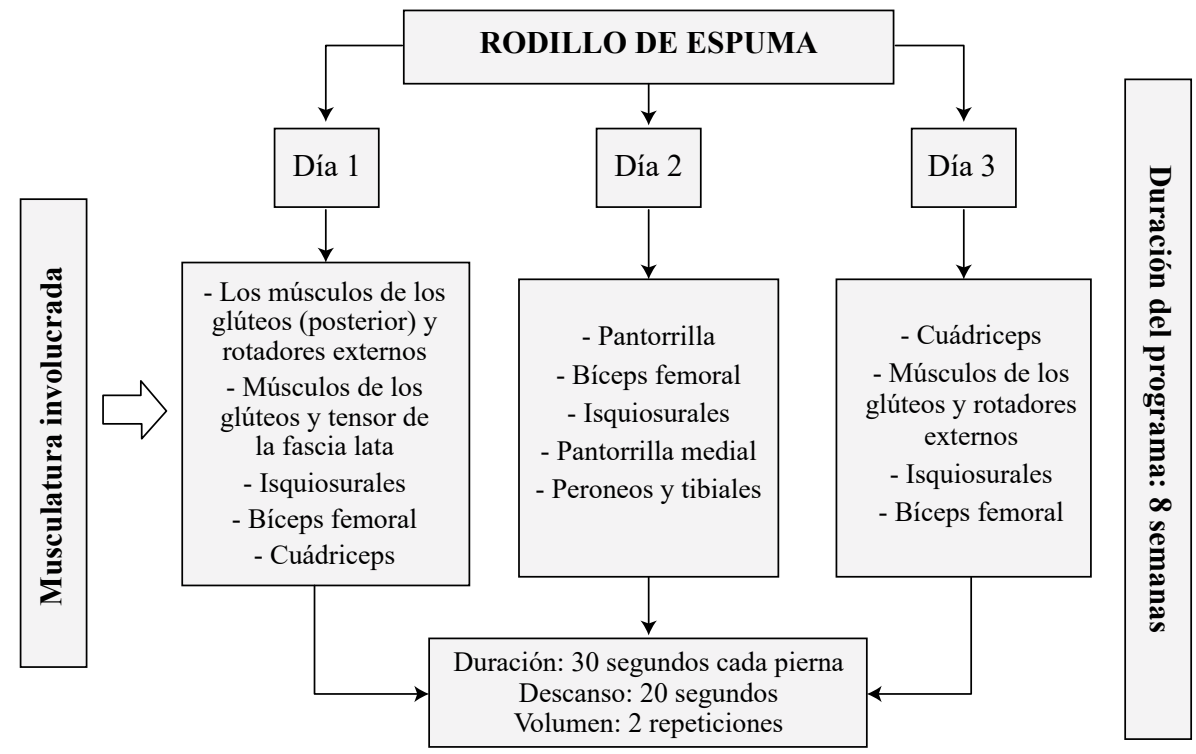

Figura 2. Plan de intervención con el rodillo de espuma (Cheatham, et al., 2015; Mohr, et al., 2014). Fuente: elaboración propia.

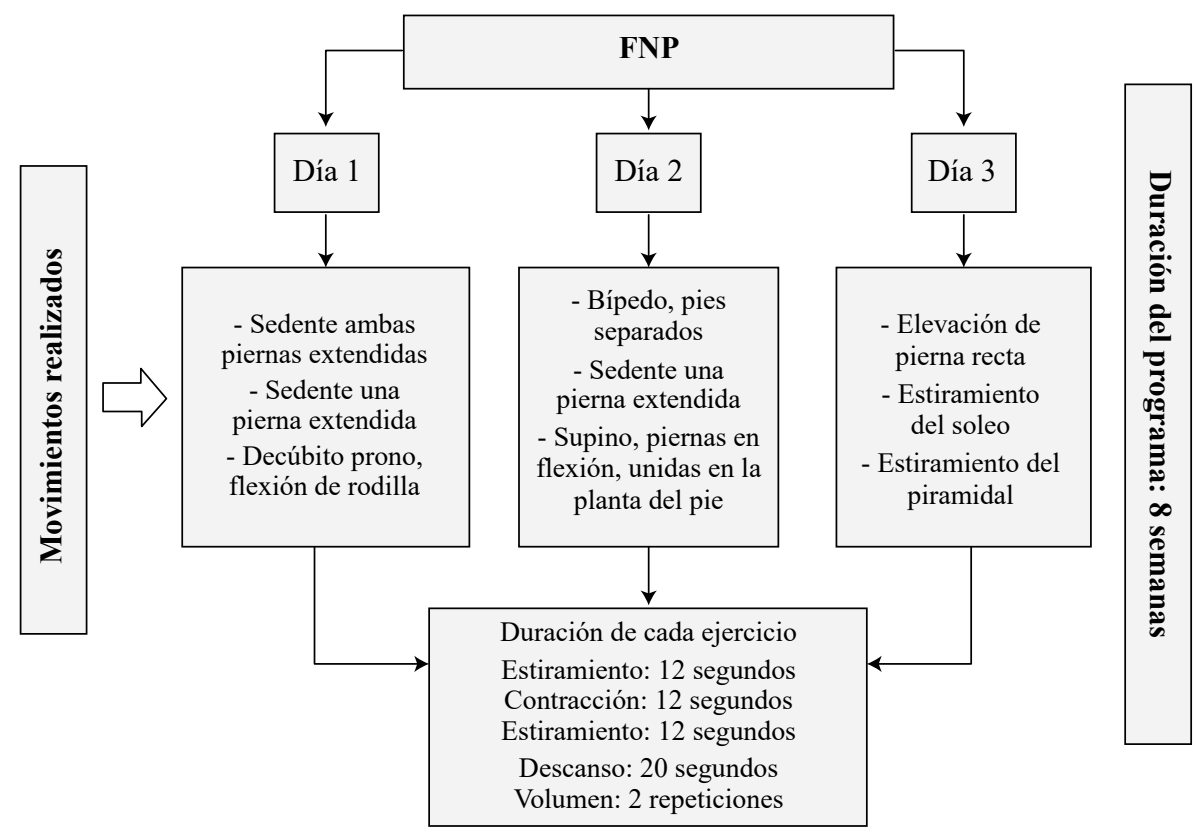

Figura 3. Plan de intervención con facilitación neuromuscular propioceptiva (FNP) (McAtee, R. \& Charland, 2007). Fuente: elaboración propia. 
grupos antes y después de desarrollar los programas de flexibilidad, y se aplicó la prueba de Scheffe para comparar las medias entre los grupos. En cuanto a las pruebas no paramétricas, se utilizó la prueba U de Mann Withney.

Para el cálculo del tamaño del efecto, se utilizó el valor de eta parcial al cuadrado y la prueba de modelo lineal generalizado (MLG) univariante para el test de flexibilidad y multivariante para la potencia de salto en términos de RMS, así como las pruebas post hoc de Scheffe y Tukey y de comparación de medias empleando el método de boostrapping.

Aspectos éticos y legales. Para el desarrollo del presente estudio se tuvieron en cuenta la Declaración de Helsinki, en la cual se establecen los principios éticos para las investigaciones médicas en seres humanos (World Medical Asociation - AMM, 2013), y la Resolución 8430 de 1993 del Ministerio de Salud de la República de Colombia sobre las normas científicas, técnicas y administrativas para la investigación en salud (Ministerio de Salud, 1993). Además, se contó con la aprobación y el aval del Comité de ética para la investigación científica expedido por la Vicerrectoría de Investigaciones Universidad Del Cauca (2015).

\section{Resultados}

Con relación a las medidas sociodemográficas y antropométricas, los participantes tenían una edad promedio de
24,35 años +/- 3,86, con un índice de masa corporal de 24,45 +/- 2,12; clasificándose como 'normopeso', con un porcentaje de grasa de $9,09+/-1,703$ y una media de la sumatoria de pliegues de 83,96+/- 22,628.

En cuanto a los antecedentes deportivos, el $39 \%$ de los participantes llevaba más de nueve años practicando fútbol sala; entrenaban cinco días a la semana $(56,5 \%)$, con un promedio de dos horas diarias por sesión de entrenamiento; el 52,2 \% de ellos jugaba en la posición de alero, y la mayoría de los futbolistas $(78,3 \%)$ utilizaban el pie derecho.

Se encontró una disminución en la sumatoria de ocho pliegues entre los grupos de rodillo de espuma y facilitación neuromuscular propioceptiva, así como una disminución en los valores de la sumatoria de pliegues de miembros inferiores del grupo de rodillo de espuma y el grupo de control después de la intervención (Tabla 1).

El grupo de rodillo de espuma registró una significación estadística menor a 0,05 y una correlación muy alta pero negativa, evidenciando que cuanto menor es el porcentaje de grasa, mayor es la altura en los saltos SJ, CMJ, ABK, unipodal derecho y unipodal izquierdo (Tabla 2).

Al comparar la altura del salto pre y post-test (figura 4) se encontró que en el grupo de rodillo de espuma con el de control, se encontró un aumento estadísticamente

Tabla 1. Variables antropométricas distribuidas por grupos de intervención

\begin{tabular}{|c|c|c|c|c|c|c|c|c|c|}
\hline \multirow[t]{3}{*}{ Variables antropométricas } & \multicolumn{9}{|c|}{ Grupos } \\
\hline & \multicolumn{3}{|c|}{ Rodillo de espuma } & \multicolumn{3}{|c|}{$\begin{array}{c}\text { Facilitación neuromuscular } \\
\text { propioceptiva }\end{array}$} & \multicolumn{3}{|c|}{ Control } \\
\hline & $\begin{array}{c}\text { Antes } \\
\text { del test }\end{array}$ & $\begin{array}{l}\text { Después } \\
\text { del test }\end{array}$ & SE & $\begin{array}{c}\text { Antes } \\
\text { del test }\end{array}$ & $\begin{array}{l}\text { Después } \\
\text { del test }\end{array}$ & SE & $\begin{array}{c}\text { Antes } \\
\text { del test }\end{array}$ & $\begin{array}{l}\text { Después } \\
\text { del test }\end{array}$ & SE \\
\hline Índice de masa corporal & 25,92 & 25,65 & 0,263 & 24,56 & 24,38 & 0,779 & 22,67 & 22,45 & 0,86 \\
\hline Sumatoria de 8 pliegues $(\mathrm{mm})$ & 97,00 & 82,25 & $0,012 *$ & 86,63 & 75,38 & $0,012 *$ & 66,00 & 65,71 & 0,92 \\
\hline $\begin{array}{l}\text { Sumatoria de pliegues muslo } \\
\text { y pantorrilla }(\mathrm{mm})\end{array}$ & 16,38 & 13,00 & $0,027 *$ & 15,38 & 13,88 & 0,078 & 13,57 & 12,43 & $0,08 *$ \\
\hline Porcentaje de grasa $(\%)$ & 9,95 & 9,81 & 0,310 & 9,31 & 8,90 & 0,122 & 7,87 & 8,08 & 0,31 \\
\hline
\end{tabular}

Fuente: Visor de resultados de SPSS, versión 23, elaboración propia

*SE: Significación estadística: p inferior a 0,05

Tabla 2. Relación entre el porcentaje de grasa y la potencia del salto en los futbolistas participantes

\begin{tabular}{lcccccc}
\hline Porcentaje de grasa & \multicolumn{2}{c}{ Rodillo de espuma } & Facilitación neuromuscular propioceptiva & \multicolumn{2}{c}{ Control } \\
\cline { 2 - 6 } & SE & Correlación & SE & Correlación & SE & Correlación \\
\hline Altura salto SJ & $\mathbf{0 , 0 0 7 *}$ & $-0,855$ & 0,690 & 0,169 & 0,760 & $-0,143$ \\
\hline Altura salto CMJ & $\mathbf{0 , 0 0 2 *}$ & $-0,905$ & 0,548 & 0,252 & 0,939 & $-0,036$ \\
Altura salto ABK & $\mathbf{0 , 0 0 0 *}$ & $-0,994$ & 0,520 & $-0,268$ & 1,000 & 0,000 \\
Altura salto pierna derecha & $\mathbf{0 , 0 0 2 *}$ & $-0,898$ & $\mathbf{0 , 0 2 0}$ & $-0,790$ & 0,294 & $-0,464$ \\
Altura salto pierna izquierda & $\mathbf{0 , 0 2 0} *$ & $-0,790$ & 0,435 & $-0,323$ & 0,310 & $-0,450$ \\
\hline
\end{tabular}

Fuente: Visor de resultados de SPSS versión 23, elaboración propia.

*SE: Significación estadística: $p$ inferior a 0,05 

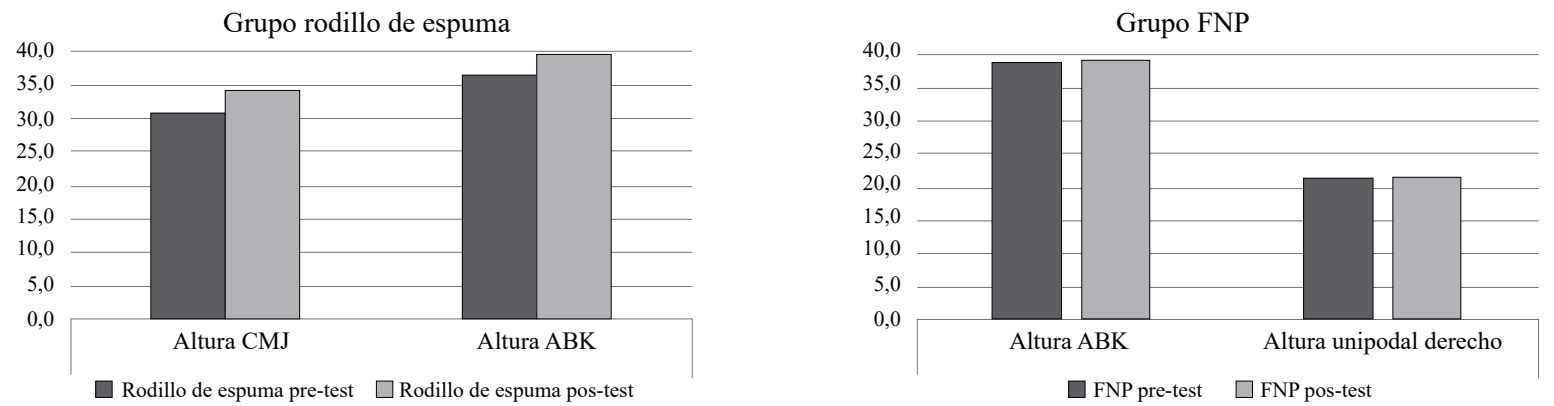

Grupo control

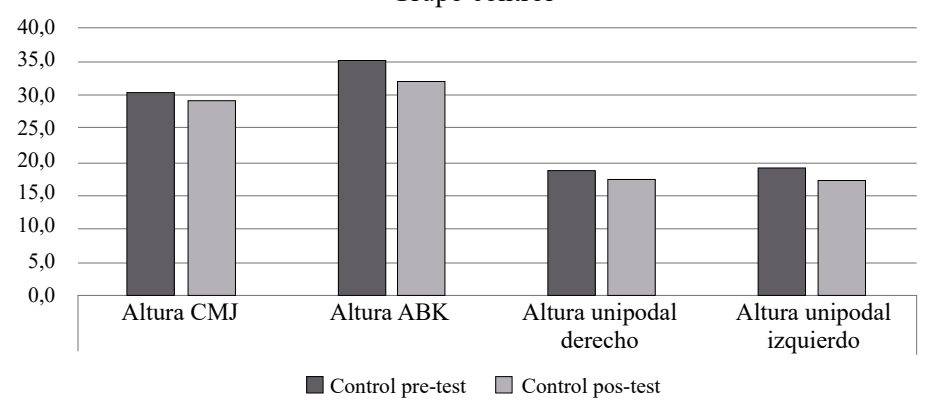

Figura 4. Comparación de la altura del salto $(\mathrm{cm})$ entre los grupos antes y después de la intervención. Fuente: Visor de resultados de SPSS versión 23, elaboración propia. Fuente: elaboración propia.

significativo en la altura del salto de contramovimiento ( $p=0,029)$, así como en la del salto Abalakov $(p=0,028)$, en tanto que con la técnica de facilitación neuromuscular propioceptiva hubo un aumento estadísticamente significativo en comparación con el grupo de control en los valores de los saltos Abalakov y unipodal derecho de $\mathrm{p}=0,042$ y $\mathrm{p}=0,048$, respectivamente; en el grupo de control se evidenció una disminución estadísticamente significativa en los resultados de los saltos de contramovimiento, Abalakov, y unipodal derecho e izquierdo $(\mathrm{p}=0,001, \mathrm{p}=0,006, \mathrm{p}=0,002$ y $\mathrm{p}=0,028$, respectivamente).

Al comparar los grupos de rodillo de espuma y facilitación neuromuscular propioceptiva con el grupo de control, en el salto en cuclillas se presentó una disminución estadísticamente significativa de los valores de la RMS de los músculos semitendinoso y bíceps femoral derecho, así como una disminución en la RMS del músculo semitendinoso izquierdo al comparar los resultados del grupo de rodillo de espuma con el grupo de control. En el salto de contramovimiento, al comparar el grupo de rodillo de espuma con el de control, se evidencio una disminución estadísticamente significativa en los valores de la RMS de los músculos semitendinoso y bíceps femoral derecho, así como en el músculo semitendinoso izquierdo, en tanto que al comparar el grupo $\mathrm{F}$ de rodillo de espuma con el de control, solo disminuyó la RMS en el músculo semitendinoso derecho. Por último, en el salto unipodal se encontró una disminución estadísticamente significativa en la RMS del músculo bíceps femoral derecho entre el grupo de facilitación neuromuscular propioceptiva y el grupo de control (Tabla 3).
Tabla 3. Comparación intergrupal de la RMS de los músculos isquiosurales

\begin{tabular}{|c|c|c|c|c|c|}
\hline Salto & $\begin{array}{l}\text { Activación } \\
\text { muscular }\end{array}$ & Grupos & & & SE \\
\hline \multirow[t]{5}{*}{ SJ } & \multirow{2}{*}{$\begin{array}{l}\text { RMS } \\
\text { semitendinoso } \\
\text { derecho }\end{array}$} & $\begin{array}{l}\text { Rodillo de } \\
\text { espuma }\end{array}$ & $V s$. & Control & 0,042 \\
\hline & & FNP & $V s$ & Control & 0,020 \\
\hline & \multirow[t]{2}{*}{$\begin{array}{l}\text { RMS bíceps } \\
\text { femoral derecho }\end{array}$} & $\begin{array}{l}\text { Rodillo de } \\
\text { espuma }\end{array}$ & $V s$ & Control & 0,032 \\
\hline & & FNP & $V s$ & Control & 0,045 \\
\hline & $\begin{array}{l}\text { RMS } \\
\text { semitendinoso } \\
\text { izquierdo }\end{array}$ & $\begin{array}{l}\text { Rodillo de } \\
\text { espuma }\end{array}$ & $V s$ & Control & 0,010 \\
\hline \multirow[t]{4}{*}{ СМJ } & \multirow{2}{*}{$\begin{array}{l}\text { RMS } \\
\text { semitendinoso } \\
\text { derecho }\end{array}$} & $\begin{array}{l}\text { Rodillo de } \\
\text { espuma }\end{array}$ & $V s$ & Control & 0,020 \\
\hline & & FNP & $V s$ & Control & 0,014 \\
\hline & $\begin{array}{l}\text { RMS bíceps } \\
\text { femoral derecho }\end{array}$ & $\begin{array}{l}\text { Rodillo de } \\
\text { espuma }\end{array}$ & $V s$. & Control & 0,023 \\
\hline & $\begin{array}{l}\text { RMS } \\
\text { semitendinoso } \\
\text { izquierdo }\end{array}$ & $\begin{array}{l}\text { Rodillo de } \\
\text { espuma }\end{array}$ & $V s$. & Control & 0,000 \\
\hline Unipodal & $\begin{array}{l}\text { RMS biceps } \\
\text { femoral derecho }\end{array}$ & FNP & $V s$ & Control & 0,045 \\
\hline
\end{tabular}

Fuente: Visor de resultados de SPSS versión 23, elaboración propia. *SE: Significación estadística: $p$ inferior a 0,05

La tabla 4 muestra que en la prueba de flexibilidad de sentarse y alcanzar, al comparar los grupos no se registró una diferencia estadísticamente significativa $(\mathrm{p}=0,072)$, sin 
Tabla 4. Tamaño del efecto en la prueba de sentarse y alcanzar y valores de la RMS después de la intervención

\begin{tabular}{|c|c|c|c|}
\hline \multicolumn{4}{|c|}{ Prueba de efectos entre sujetos - Flexibilidad en la prueba de sentarse y alcanzar } \\
\hline & & SE & Eta parcial al cuadrado \\
\hline Interceptación & & 0,000 & 0,556 \\
\hline Grupo & & 0,072 & 0,231 \\
\hline \multicolumn{4}{|c|}{ Comparación por parejas } \\
\hline (I) & (J) & SE & Diferencia de medias (I-J) \\
\hline \multirow{2}{*}{$\begin{array}{c}\text { Control } \\
(2,314 \pm 6,7952)\end{array}$} & Rodillo de espuma $(7,375 \pm 8,0345)$ & 0,214 & $-5,0607$ \\
\hline & $\operatorname{FNP}(10,375 \pm 3,5431)$ & 0,026 & $-8,0607$ \\
\hline \multirow[t]{3}{*}{$\begin{array}{c}\text { FNP } \\
(10,375 \pm 3,5431)\end{array}$} & Rodillo de espuma $(7,375 \pm 8,0345)$ & 0,350 & 3,0000 \\
\hline & \multicolumn{3}{|c|}{ Prueba de efectos inter-sujetos - RMS en los saltos (CMJ, SJ, ABK) } \\
\hline & & SE & Eta parcial al cuadrado \\
\hline Interceptación & & 0,000 & 0,996 \\
\hline Grupo & & 0,206 & 0,840 \\
\hline
\end{tabular}

Prueba post hoc de comparaciones múltiples

(I)

(J) SE

RMS salto SJ músculo semitendinoso derecho, final

\begin{tabular}{|c|c|c|c|}
\hline (I) & (J) & SE & Diferencia de medias (I-J) \\
\hline \multicolumn{4}{|c|}{ RMS salto SJ músculo semitendinoso derecho, final } \\
\hline \multirow{2}{*}{$\begin{array}{l}\text { Rodillo de espuma } \\
(0,15263 \pm 0,45663)\end{array}$} & FNP & 0,922 & 0,01263 \\
\hline & Control & $\mathbf{0 , 0 3 3}$ & $-0,09295^{*}$ \\
\hline \multirow{2}{*}{$\begin{array}{c}\text { FNP } \\
(0,14000 \pm 0,61894)\end{array}$} & Rodillo de espuma & 0,922 & $-0,01263$ \\
\hline & Control & 0,015 & $-0,10557^{*}$ \\
\hline \multirow{2}{*}{$\begin{array}{c}\text { Control } \\
(0,24557 \pm 0,86835)\end{array}$} & Rodillo de espuma & $\mathbf{0 , 0 3 3}$ & $0,09295^{*}$ \\
\hline & FNP & 0,015 & $0,10557^{*}$ \\
\hline \multicolumn{4}{|c|}{ RMS salto SJ músculo bíceps femoral derecho final } \\
\hline \multirow{2}{*}{$\begin{array}{l}\text { Rodillo de espuma } \\
(0,14663 \pm 0,47656)\end{array}$} & FNP & 0,985 & $-0,00450$ \\
\hline & Control & 0,025 & $-0,07952^{*}$ \\
\hline \multirow{2}{*}{$\begin{array}{c}\text { FNP } \\
(0,15113 \pm 0,038432)\end{array}$} & Rodillo de espuma & 0,985 & 0,00450 \\
\hline & Control & $\mathbf{0 , 0 3 5}$ & $-0,07502^{*}$ \\
\hline \multirow{2}{*}{$\begin{array}{c}\text { Control } \\
(0,22614 \pm 0,072474)\end{array}$} & Rodillo de espuma & 0,025 & $0,07952^{*}$ \\
\hline & FNP & $\mathbf{0 , 0 3 5}$ & $0,07502^{*}$ \\
\hline \multicolumn{4}{|c|}{ RMS salto SJ músculo semitendinoso izquierdo final } \\
\hline \multirow{2}{*}{$\begin{array}{l}\text { Rodillo de espuma } \\
(0,12525 \pm 0,033281)\end{array}$} & FNP & 0,341 & $-0,03312$ \\
\hline & Control & 0,007 & $-0,08204^{*}$ \\
\hline \multirow{2}{*}{$\begin{array}{c}\text { FNP } \\
(0,15838 \pm 0,042251)\end{array}$} & Rodillo de espuma & 0,341 & 0,03312 \\
\hline & Control & 0,126 & $-0,04891$ \\
\hline \multirow{2}{*}{$\begin{array}{c}\text { Control } \\
(0,20729 \pm 0,060802)\end{array}$} & Rodillo de espuma & $\mathbf{0 , 0 0 7}$ & $0,08204^{*}$ \\
\hline & FNP & 0,126 & 0,04891 \\
\hline \multicolumn{4}{|c|}{ RMS salto CMJ músculo semitendinoso derecho final } \\
\hline \multirow{2}{*}{$\begin{array}{l}\text { Rodillo de espuma } \\
(0,15650 \pm 0,039123)\end{array}$} & FNP & 0,981 & 0,00575 \\
\hline & Control & 0,015 & $-0,09779^{*}$ \\
\hline \multirow{2}{*}{$\begin{array}{c}\text { FNP } \\
(0,15075 \pm 0,065939)\end{array}$} & Rodillo de espuma & 0,981 & $-0,00575$ \\
\hline & Control & $\mathbf{0 , 0 1 0}$ & $-0,10354^{*}$ \\
\hline
\end{tabular}

Diferencia de medias (I-J) 


\begin{tabular}{|c|c|c|c|}
\hline \multirow{2}{*}{$\begin{array}{c}\text { Control } \\
(0,25429 \pm 0,074819)\end{array}$} & Rodillo de espuma & 0,015 & $0,09779^{*}$ \\
\hline & FNP & $\mathbf{0 , 0 1 0}$ & $0,10354^{*}$ \\
\hline \multicolumn{4}{|c|}{ RMS salto CMJ músculo bíceps femoral derecho final } \\
\hline \multirow{2}{*}{$\begin{array}{l}\text { Rodillo de espuma } \\
(0,14213 \pm 0,037177)\end{array}$} & FNP & 0,689 & $-0,02050$ \\
\hline & Control & $\mathbf{0 , 0 1 7}$ & $-0,07745^{*}$ \\
\hline \multirow{2}{*}{$\begin{array}{c}\text { FNP } \\
(0,16263 \pm 0,043474)\end{array}$} & Rodillo de espuma & 0,689 & 0,02050 \\
\hline & Control & 0,091 & $-0,05695$ \\
\hline \multirow{2}{*}{$\begin{array}{c}\text { Control } \\
(0,21957 \pm 0,065653)\end{array}$} & Rodillo de espuma & $\mathbf{0 , 0 1 7}$ & $0,07745^{*}$ \\
\hline & FNP & 0,091 & 0,05695 \\
\hline \multicolumn{4}{|c|}{ RMS salto CMJ musculo semitendinoso izquierdo final } \\
\hline \multirow{2}{*}{$\begin{array}{l}\text { Rodillo de espuma } \\
(0,12675 \pm 0,033457)\end{array}$} & FNP & 0,048 & $-0,04275^{*}$ \\
\hline & Control & $\mathbf{0 , 0 0 0}$ & $-0,08582^{*}$ \\
\hline \multirow{2}{*}{$\begin{array}{c}\text { FNP } \\
(0,16950 \pm 0,033076)\end{array}$} & Rodillo de espuma & 0,048 & $0,04275^{*}$ \\
\hline & Control & 0,055 & $-0,04307$ \\
\hline \multirow{2}{*}{$\begin{array}{c}\text { Control } \\
(0,21257 \pm 0,034253)\end{array}$} & Rodillo de espuma & $\mathbf{0 , 0 0 0}$ & $0,08582^{*}$ \\
\hline & FNP & 0,055 & 0,04307 \\
\hline \multicolumn{4}{|c|}{ RMS salto pierna derecha musculo bíceps femoral final } \\
\hline \multirow{2}{*}{$\begin{array}{l}\text { Rodillo de espuma } \\
(0,22325 \pm 0,074133)\end{array}$} & FNP & 0,885 & 0,01875 \\
\hline & Control & 0,089 & $-0,09204$ \\
\hline \multirow{2}{*}{$\begin{array}{c}\text { FNP } \\
(0,20450 \pm 0,49179)\end{array}$} & Rodillo de espuma & 0,885 & $-0,01875$ \\
\hline & Control & $\mathbf{0 , 0 3 5}$ & $-0,11079^{*}$ \\
\hline \multirow{2}{*}{$\begin{array}{c}\text { Control } \\
(0,31529 \pm 0,108465)\end{array}$} & Rodillo de espuma & 0,089 & 0,09204 \\
\hline & FNP & $\mathbf{0 , 0 3 5}$ & $0,11079^{*}$ \\
\hline
\end{tabular}

*SE. Significación estadística

embargo, la eta parcial al cuadrado tuvo un valor de 0,231 , lo que indica que el tamaño del efecto fue moderado. El valor de la interceptación evidenció que estadísticamente hubo una diferencia significativa entre los grupos, con un valor de $\mathrm{p}=0,000$ y un valor de eta parcial al cuadrado de 0,556 , lo que da cuenta de un tamaño del efecto marcado, por lo que al comparar mediante la prueba post hoc de Scheffe, se encontró una diferencia estadísticamente significativa entre los grupos de control y de facilitación neuromuscular propioceptiva, con una significación de $\mathrm{p}=0,026$ (aplicando un procedimiento de boostrapping).

Asimismo, los valores de la RMS en la comparación general de los grupos no reflejaron una diferencia estadísticamente significativa $(\mathrm{p}=0,206)$, sin embargo, el eta parcial al cuadrado registró un valor de 0,840 , lo que indica que el tamaño del efecto fue marcado. El valor de la interceptación evidenció que estadísticamente hubo una diferencia significativa entre los grupos, con un valor de $\mathrm{p}=0,000$ y un valor de eta parcial al cuadrado de 0,996 , es decir, se registró un marcado tamaño del efecto, por lo que, al comparar mediante la prueba post hoc de Tukey con un procedimiento de boostrapping, se encontró una diferencia estadísticamente significativa entre el grupo de control y los grupos de facilitación neuromuscular propioceptiva y de rodillo de espuma para la mayoría de variables, excepto las de "RMS salto CMJ músculo bíceps femoral izquierdo final"; "RMS salto ABK músculo semitendinoso derecho final"; "RMS salto ABK músculo bíceps femoral derecho final; "RMS salto ABK músculo bíceps femoral izquierdo final"; RMS salto pierna derecha músculo semitendinoso final", y "RMS salto pierna izquierda musculo bíceps femoral final" ( $p>0,05)$.

\section{Discusión}

Las características sociodemográficas y físicas de una población de deportistas varían según diversos factores como el perfil genético, el período del entrenamiento, el nivel competitivo y la edad de los jugadores. Sebastia-Amat, et al. (2017) y Matzenbacherab, et al. (2014) plantean que la estatura y el peso corporal de los deportistas de fútbol sala es una variable determinante en el rendimiento deportivo: la estatura debe estar entre $172,8 \pm 5,5$ y $184 \pm 2 \mathrm{~cm}$ y el peso, entre $68,5 \pm 9,5 \mathrm{~kg}$ y $85,9 \pm 10,2 \mathrm{~kg}$, lo que sugiere que la población evaluada en el presente estudio presentó una talla y un peso por debajo del promedio sugerido para la práctica de este deporte, aunque el IMC promedio, de 24,4 $\pm 2,1$, sí 
se encuentra dentro de los rangos sugeridos por diferentes autores (Cecil, et al., 2017; Avelar, et al., 2008).

Con relación al porcentaje de grasa y la potencia del salto, se encontró que la significación estadística entre estas dos variables y la correlación negativa fueron muy altas en el grupo de rodillo de espuma; es decir, que a menor porcentaje de grasa, mayor la altura en los saltos, tal como lo describen Rendón-Morales, et al. (2017) y Sebastiá-Amat, et al. (2017), quienes concluyen que cuanta más alta es la cantidad de masa grasa presente en el atleta, menor es el rendimiento del salto vertical, variable que se puede ver afectada por el estilo de vida y la nutrición del deportista. Asimismo, se debe tener en cuenta que la relación entre el porcentaje de grasa y las capacidades físicas fluctúa en los deportistas, ya que durante los periodos preparatorios o competitivos las capacidades funcionales de los atletas tienden a aumentar (Fuke, et al., 2009).

$\mathrm{Al}$ analizar los cambios en la altura del salto en el grupo de rodillo de espuma, se observó un aumento significativo antes y después de la intervención en los saltos de contramovimiento y Abalakov, lo cual se debe a que este método de estiramiento no solo mejora la liberación de adherencias musculares, sino también la capacidad de alargamiento y la velocidad de contracción de las fibras musculares (Lavandero, et al., 2017). Además, el uso del rodillo de espuma comprado con otras técnicas de estiramiento mejora de manera patente el potencial, la velocidad y la agilidad en las pruebas de rendimiento (Peacock, et al., 2014). Asímismo, el rodillo de espuma mejora sustancialmente la sensibilidad del músculo, mejorando la capacidad de aceleración, la potencia y la resistencia a la fuerza del músculo (Pearcey, et al., 2015). Sin embargo, cabe aclarar que una de las limitaciones que presenta el estudio fue que no se consideraron las cargas de entrenamiento realizadas por el entrenador para variables como la fuerza y la velocidad, capacidades que pueden influir en los resultados, lo cual debe tenerse en cuenta en próximos estudios en este tema.

En este mismo sentido, los integrantes del grupo de facilitación neuromuscular propioceptiva presentaron un aumento significativo en la altura de los saltos Abalakov y unipodal derecho, mientras que el grupo de control presentó una disminución en los resultados de la evaluación posterior a la intervención. Esto se debe, en gran medida, a que la técnica de facilitación neuromuscular propioceptiva incorpora la contracción y la relajación muscular en posiciones de amplitud articular máxima, generando un mayor estímulo propioceptivo, reduciendo los niveles de actividad eléctrica y aumentando la sensación de relajación muscular (Da Rocha-Mafra, et al., 2013).

Es importante anotar que en los saltos de contramovimiento, Abalakov y unipodales se produjeron los cambios más significativos; estos movimientos requieren de una gran aceleración e incrementan la eficacia mecánica y la potencia del músculo. Neurofisiológicamente esto se puede explicar gracias a la activación del reflejo miotático, ya que el estiramiento rápido de un grupo muscular produce un aumento en la acumulación de energía elástica, facilitando el aumento de la potencia durante la posterior contracción concéntrica, lo que se denomina como ciclo de estiramientoacortamiento (Gutiérrez-Dávila, et al., 2015)

La cuantificación de la señal eléctrica refleja la actividad fisiológica en la unidad motora durante la contracción muscular, conocida como RMS (Yukio-Fukuda, et al., 2010) vastus medialis, lateralis, biceps femoris, semitendinosus, and brachial biceps muscles. Methods: The analysis was performed on 24 females, university students with a mean age of $20( \pm 6$. En este estudio los valores en la evaluación posterior disminuyeron para los grupos experimentales, en tanto que en el grupo de control se registró un aumento en los valores de la RMS después de la intervención comparados con las evaluaciones iniciales. Esto se debe, en gran parte, a que las técnicas de estiramiento utilizadas con el rodillo de espuma y de facilitación neuromuscular propioceptiva mejoran el ciclo de estiramiento - acortamiento, activando el reflejo miotático, lo cual ayuda a aprovechar la energía elástica, es decir, la capacidad que tiene el músculo para almacenar energía durante el estiramiento y utilizarla en una posterior contracción o acción de potencia (BelmonteFérez, 2015). Por esta razón, los valores de la RMS disminuyeron después de la intervención, ya que los músculos isquiosurales necesitaron una menor activación para generar una actividad explosiva, aumentando la potencia media de los músculos involucrados en el salto vertical sin ocasionar un mayor gasto energético (Rousanoglou, et al., 2013).

Es claro que el uso del rodillo de espuma, en comparación con la aplicación de la técnica de facilitación neuromuscular propioceptiva, genera mayores cambios electromiograficos. Button, et al. (2014) utilizaron en su estudio el rodillo de espuma en actividades de 20 a 60 segundos y analizaron el rango de movimiento en la articulación de la rodilla y su efecto durante la estocada, encontrando que su uso aumentó de manera significativa la amplitud de movimiento de la articulación y la eficiencia neuromuscular durante la estocada, lo que se reflejó en una disminución del promedio del valor de la RMS a medida que aumentaba el uso del masajeador o rodillo de espuma. Esto concuerda con los resultados del presente estudio, pero, por el contrario, estos autores encontraron un incremento significativo en el arco de movilidad articular, sin cambios importantes en los valores electromiograficos después de usar el rodillo de espuma durante actividades de 5 y 10 segundos, lo cual indica que para que su uso disminuya los valores de la RMS se debe aplicar por más de 20 segundos.

\section{Conclusiones}

Cuando el índice de masa corporal y el porcentaje de grasa aumentan sus valores, estos inciden negativamente en la potencia del salto, situación que debe ser tenida en cuenta para el control del rendimiento deportivo y la valoración médica y física de los futbolistas. 
Tanto el rodillo de espuma como la facilitación neuromuscular propioceptiva parecen ser eficaces en el mejoramiento de la potencia del salto después de una intervención de ocho semanas con un estímulo semanal de tres veces por semana de entrenamiento de la musculatura isquiosural.

El comportamiento muscular durante el salto después de una intervención de entrenamiento de la flexibilidad de la musculatura isquiosural parece ser efectiva para mejorar la capacidad de reclutamiento motor, facilitando una menor activación con mayor rendimiento de la fibra muscular para contraerse y generar una contracción rápida y fuerte que se traduce en un movimiento más potente y favorece el rendimiento deportivo del futbolista.

Por último, en este estudio se registraron resultados favorables al entrenar la flexibilidad de manera planificada y estructurada a partir de dos técnicas de estiramiento, las cuales pueden ser utilizadas por los entrenadores o fisioterapeutas durante los procesos de entrenamiento puara mejorar el rendimiento deportivo. Por otra parte, se sugiere continuar con la investigación sobre la relación de estas dos variables en el rendimiento del atleta con un mayor número de participantes o con otras técnicas de estiramiento que puedan brindar información para mejorar los procesos de entrenamiento en el fútbol sala.

\section{Agradecimientos}

Agradecemos a la Universidad del Cauca, al programa de Maestría en Deporte y Actividad Física y al Club Atlético Cauca, por permitirnos realizar este proyecto.

\section{Contribución de los autores}

Enmanuel Portilla-Dorado: elaboración base de datos, análisis estadístico, redacción del artículo y autor de correspondencia. Andrés Villaquiran-Hurtado: diseño del estudio, recolección de los datos, redacción del artículo. Nancy Jannet Molano-Tobar: revisión de la literatura, trabajo de campo, análisis de resultados, redacción del artículo.

\section{Conflicto de interereses}

Los autores declaran no tener ningun conflicto de intereses.

\section{Referencias}

Alberto, M. G., Lavaho, M., Milena, S. (2016). Percepción del esfuerzo en entrenamiento de futbolistas categoría sub 15. Revista Edu-Fisica.Com Ciencias Aplicadas al Deporte. 8: 43-75.

Álvarez-Pérez, P. R., \& López-Aguilar, D. (2018). Modelo explicativo sobre trayectorias vitales y desarrollo vocacional en deportistas de alto nivel. Revista Retos. 34: 51-56 ( $2^{\circ}$ semestre).

Aniotz, A. F., Ramírez, S. A., Guzmán, R., Espinoza, R. M. (2015). Effect of a plyometric training program on jumping biomechanics in female youth volleyball players. Revista Ciencias de la Actividad Física UCM. 16 (1): 37-44.

Arins, F. B., Carminatti, L. J., Salvador, P. C. do N., Guglielmo, L. G. A. (2015). Physiological characteristics, evaluation and prescription of aerobic training in Futsal.
Rev. Brasileira de Cineantropometria e Desempenho Humano Desempenho Humano. 17 (6): 753-762. Doi: 10.5007/1980-0037.2015v17n6p753

Avelar, A., Dos santos, K., Cyrino, E. S., Carvalho, F. O., Ritti Dias, R. M., Gobbo, L. A. (2008). Perfil antropométrico e de desempenho motor de atletas paranaenses de futsal de elite. Revista Brasileira de Cineantropometria \& Desempenho Humano. 10 (1): 76-80.

Ayala, F., Sainz-de Baranda, P., Cejudo, A., De Ste. Croix, M. (2010). Efecto de un programa de estiramientos activos en jugadoras de fútbol sala de alto rendimiento. Ciencia, Cultura y Deporte. 5 (15): 159-167. https://doi.org/10.12800/ccd. v5i15.105

Ayala, F., Sainz-de Baranda, P., De Ste Croix, M., Santonja, F. (2012). Reproducibility and criterion-related validity of the sit and reach test and toe touch test for estimating hamstring flexibility in recreationally active young adults. Physical Therapy in Sport. 13 (4): 219-226. Doi: 10.1016/j. ptsp.2011.11.001

Belmonte-Férez, J. D. (2015). Efectos de un programa de estiramientos FNP en el salto vertical y el rango de movimiento en futbolistas profesionales. RediUMH Universidad Miguel Hernández. 7 (21): 1-10.

Bonjour, L. (2017). El foam roller como herramienta de prevencion de la lesion de isquiotibiales en futbolistas amateurs. Universidad Pública de Navarra. 1-103.

Bosco, C., Luhtanen, P., Komi, P. V. (1983). A simple method for measurement of mechanical power in jumping. European Journal of Applied Physiology and Occupational Physiology. 50 (2): 273-282. Doi: 10.1007/BF00422166

Button, D. C., Bradbury-squired, D., Noftall, J., Sullivan, K., Behm, D. G., Power, K. (2014). Roller-Massager Application to the Quadriceps and Knee-Joint Range of Motion and Neuromuscular Efficiency During a Lunge. Journal of Athletic Training. 49 (5): 133-140. Doi: 10.4085/10626050-49.5.03

Carminatti, L. J., Arins, F. B., Silva, J. F., Santos, P. C., Silva, C. E. M., Guglielmo, L. G. A. (2015). Intensidade de esforço em jogos oficiais e simulados de futsal feminino. Revista Brasileira de Ciência e Movimento. 23 (3): 97-104.

Castañer, M., Saüch, G., Camerino, O., Sánchez-Algarra, P., Anguera, M. T. (2015). Percepción de la intensidad al esfuerzo: Un estudio multi-method en actividad física. Cuadernos de Psicología Del Deporte. 15 (1): 83. Doi: 10.4321/S1578-84232015000100008

Cecil, F., Lima, T., Mineiro, A., Guedes, D., Scorcine, C., Madureira, F., Pereira, R. (2017). Caracteristicas fisiologicas e antropometricas de atletas profissionais de futebol e futsal. Revista Brasileira de Futsal e Futebol. 9 (32): 21-26.

Cheatham, S. W., Kolber, M. J., Cain, M., Lee, M. (2015). The effects of self-myofascial release using a foam roll or roller massager on joint range of motion, muscle recovery, and performance: a systematic review. International Journal of Sports Physical Therapy. 10 (6): 827-838. Doi: 10.12968/ ijtr.2014.21.12.569

Da Rocha-Mafra, O., Soares, J., Alves de Moraes Filho, J., Chulvi-Medrano, I., Sánchez-Colado, J. C., MartínDantas, E. H. (2013). Análisis electromiográfico del efecto de relajación en el bíceps femoral en sujetos sometidos a estiramientos vs. facilitación neuromuscular propioceptiva. Fisioterapia. 35 (2): 47-51. Doi: 10.1016/j.ft.2012.05.007 
Dal Pupo, J., Detanico, D., Arins, F. B., Nascimento, P. C., Guglielmo, L. G. A., Dos Santos, S. G. (2016). Capacidade de sprints repetidos e níveis de potência muscular em jogadores de futsal das categorias sub-15 e sub-17. Revista Brasileira de Ciências Do Esporte. 39, n.1, pp.73-78_Vol.?: Doi: 10.1016/j.rbce.2016.01.010

Departamento Administrativo del Deporte, la Recreación, la Actividad Física y el Aprovechamiento del Tiempo Libre - Coldeportes. (2015). Lineamientos de Política Pública en Ciencias del Deporte en Medicina. Bogotá, D.C,Tiraje de 1 a 1.400, Printed in Colombia, ISBN: 978-958-58238-3-9

Foster, C. (1998). Monitoring training in athletes with reference to overtraining syndrome. Medicine \& Sscience in Sports \& Exercise. 30. (7). 1164-1168.

Fuke, K., Dal Pupo, J., Matheus, S. C. (2009). Evaluación de la composición corporal y de la flexibilidad en futbolistas profesionales en diferentes etapas del ciclo de entrenamiento. Archivos de Medicina Del Deporte. 26 (129): 7-13.

Gutiérrez-Dávila, M., Javier, F., Girela, G., Ropero, C. G., Javier, F., Ruiz, R. (2015). Efecto de la intensidad del contramovimiento sobre el rendimiento del salto vertical. Apunts. Educación Física y Deporte. 1 (119): 87-96.

Gutiérrez-Manzanedo, J. V., Del Rosario-Fernández Santos, J., Ponce Gónzalez, J. G., Lagares-Franco, C., De Castro Maqueda, G. R. (2018). Extensibilidad isquiosural en jugadoras de élite de fútbol. (33): 175-178. consultado 5 de febrero de 2019, Consultado en: http:/ezproxy.eafit. edu.co/login?url=http://search.ebscohost.com/login.aspx?d irect $=$ true $\& \mathrm{db}=$ fua $\& A N=126604340 \&$ lang $=$ es \& site $=$ edslive \&scope $=$ site

Hermens, H. J., Freriks, B., Merletti, R., Stegeman, D., Blok, J., Rau, G., ... Hägg, G. (1999). European Recommenda-tions for Surface ElectroMyoGraphy. Roessingh Research and Development. 10: 8-11. Doi: 10.1016/S1050-6411(00)00027-4

Lavandero, G. C., Anthony, P., Morales, R., Fabián, E., Analuiza, A., Santiago, E., ... Gibert, A. R. (2017). Efectos de la autoliberación miofascial. Revisión sistemática. Revista Cubana de Investigaciones Biomédicas. 36 (2): 271-283.

López, F. Lara, A. Espejo, N., Cachón, J. (2015). Evaluación de la fuerza explosiva de extensión de las extremidades inferiores en escolares. Apunts. Educación Física y Deportes. 4 (122): 44-51. Doi: http://dx.doi.org/10.5672/ apunts.2014-0983.es.(2015/4).122.05

Luarte, C., González, M., Aguayo, O. (2014). Evaluacion de la fuerza de salto vertical en voleibol femenino en relacion a la posicion de juego. Revista Ciencias de La Actividad Fisica UCM. 15 (2): 43-52.

Matzenbacherab, F., Pasquarelle, B. N., Rabelo, F. N. L., Stanganelli, C. R. (2014). Medicina del Deporte Demanda fisiológica no futsal competitivo. Revista Andaluza de Medicina Del Deporte. 7 (3): 122-131.

McAtee, R., Charland, J. (2007). Estiramientos Facilitados. Estiramientos y fortalecimiento con facilitacion nuromuscular propioceptiva. Edición: $3^{\text {a }}$ Especialidad: Fisioterapia. Pp 212.

Ministerio de Salud. (1993). Resolución 8430 de 1993. Ministerio de Salud y Protección Social, República de Colombia, 1993. Octubre 4: 1-19.

Mohr, A. R., Long, B. C., Goad, C. L. (2014). Effect of Foam Rolling and Static Stretching on Passive Hip-Flexion Range of Motion. Journal of Sport Rehabilitation. 23 (4): 296-299. Doi: 10.1123/JSR.2013-0025
Myer, G. D., Ford, K. R., Hewett, T. E. (2005). The effects of gender on quadriceps muscle activation strategies during a maneuver that mimics a high ACL injury risk position. Journal of Electromyography and Kinesiology. 15 (2): 181-189. Doi: 10.1016/j.jelekin.2004.08.006

Nelson, A. G., Kokkonen, J., Cornwell, A. (2013). Acute muscle stretching inhibits maximal strength performance. Research Quarterly for Exercise and Sport. 69 (4): 411-415. Doi: 10.1080/02701367.2001.10608978

Ogura, Y. Miyahara, Y. Naito, H. Katamoto, S., Aoki, J. (2007). Duration of static stretching influences muscle force production in hamstring muscles. Journal of Strength and Conditioning Research. 21 (3): 788-792. Doi: 10.1519/R19535.1

Peacock, C. A., Krein, D. D., Silver, T. A., Sanders, G., KylePatrick, V. C. (2014). An Acute Bout of Self-Myofascial Release in the Form of Foam Rolling Improves Performance Testing. International Journal of Exercise Science. 7 (3): 202-211.

Pearcey, G., Bradbury-squires, D., Kawamoto, J.-E., Drinkwater, E. J., Behm, D. G., Button, D. C. (2015). Foam Rolling for Delayed-Onset Muscle Soreness and Recovery of Dynamic Performance Measures. Journal of Athletic Training. 50 (1): 5-13. Doi: 10.4085/1062-605050.1 .01

Picabea, J. \& Yanci, J. (2015). Diferencias entre jugadores de futbol, baloncesto y tenis de mesa en la capacidad de salto vertical y horizontal. Revista Iberoamericana de Ciencias de La Actividad Fisica y El Deporte. 4 (2): 9-26.

Rendón-Morales, P. A., Lara-Chalá, L. del R., Hernández, J. J., Alomoto-Navarrete, M. R., Landeta-Valladares, L. J., Calero-Morales, S. (2017). Influencia de la masa grasa en el salto vertical de basquetbolistas de secundaria. Revista Cubana de Investigaciones Biomedicas. 36 (1): 1-13. http://scielo.sld.cu/scielo.php?script=sci_arttext\&pid $=$ S0864-03002017000100015

Rodrigues Santa Cruz, R. A., Diniz Campos, F. A., Branco Gomes, I. C., Pellegrinotti, Í.L. (2016). Percepção subjetiva do esforço em jogos oficiais de Futsal. / Perception subjective effort in official Futsal games. Revista Brasileira de Ciência e Movimento: RBCM. 24 (1): 80-85. http:// search.ebscohost.com/login.aspx?direct $=$ true $\& \mathrm{db}=\mathrm{s} 3 \mathrm{~h} \& \mathrm{~A}$ $\mathrm{N}=114631315$ \&lang=pt-br\&site=ehost-live

Rodríguez, I. (2016). Valoración de la composición corporal por antropometría y bioimpedancia eléctrica. trabajo de fin de grado, Universidad Francisco de Vitoria, Madrid, Pp: 73

Rousanoglou, E. N., Barzouka, K., Boudolos, K. (2013). Seasonal changes of jumping performance and knee muscle strength in under-19 women volleyball players. Journal of Strength and Conditioning Research. 27 (4): 1108-1117.

Ruiz, H. (2013). Caracterizacion de la potencia en miembro inferior de jugadores de la seleccion de voleibol de la universidad del valle (tesis de pregrado) Universidad del Valle. Colombia.

Sainz-de Baranda-Andújar, P. \& Ayala, F. (2010). Efecto agudo del estiramiento sobre la agilidad y coordinación de movimientos rápidos en jugadores de fútbol de División de Honor. Kronos. Revista Cientifica de Actividad Fisica y Deporte. 17 (1): 21-28.

Sainz-de Baranda, P., Cejudo, A., Ayala, F. (2012). Fiabilidad absoluta del test de elevación de la pierna recta en jugadores de fútbol sala. Revista Kronos, rendimiento en deporte. 11 (I): $30-35$. 
Santiago, A., Granados, C., Quintela, K., Irigoyen, J. Y. (2015). Diferencias entre jugadores de fútbol de distintas edades en la capacidad de aceleración, cambio de dirección y salto. Cultura, Ciencia y Deporte. 10 (29): 135-143. Doi: 10.12800/ccd.v10i29.551

Sebastiá-Amat, S., Espina-Agullo, J. J., Chinchilla-Mira, J. J. (2017). Perfil de salto vertical, velocidad, flexibilidad y composición corporal de porteros de balonmano en categorías inferiores. Retos. Nuevas Tendencias En Educación Física, Deporte y Recreación. 32: 248-251.

Stewart, A., Marfell-Jones, M., Olds, T., de Ridder, H. (2011). Protocolo Internacional para la Valoración Antropométrica (2011). ISBN 0868037125

Vicerrectoría de Investigaciones - Universidad Del Cauca. (2015). Marco ético legal, Código: PM-IV-6.1-OD-2 Versión: 1, Popayan - Colombia. Pp: 1-23.
Woolstenhulme, M. T., Griffiths, C. M., Woolstenhulme, E. M., Parcell, A. C. (2006). Ballistic stretching increases flexibility and acute vertical jump height when combined with basketball activity. Journal of Strength and Conditioning Research / National Strength \& Conditioning Association. 20 (4): 799-803. Doi: 10.1519/R-18835.1

World Medical Asociation (AMM). (2013). Declaración de Helsinki de la AMM - Principios éticos para las investigaciones médicas en seres humanos. Clinical Review \& Education. 310 (20). Doi: 10.1001/jama.2013.281053

Yukio-Fukuda, T., Oliveira-Echeimberg, J., Pompeu, J. E., García-Lucareli, P. R., Garbelotti, S., Okano-Gimenes, R., Apolinário, A. (2010). Root mean square value of the electromyographic signal in the isometric torque of the quadriceps, hamstrings and brachial biceps muscles in female subjects. The Journal of Applied Research. 10 (1): 32-39. 